\title{
Synthetic Biology for Teaching Control Engineering: A Case Study in a Student-Directed, Collaborative Environment
}

\author{
Christian Euler ${ }^{l}$ and Radhakrishnan Mahadevan \\ Laboratory of Metabolic Systems Engineering, Department of Chemical Engineering and Applied Chemistry, University \\ of Toronto \\ ${ }^{1}$ Corresponding author: christian.euler@mail.utoronto.ca
}

\begin{abstract}
As the bio-based economy expands, Chemical Engineering graduates will find themselves in new contexts for which they must be prepared. The broad shift toward including biology in departmental research and teaching activities reflects this, but relatively little formal thought has been given to the pedagogy of biology within Chemical Engineering curricula. The case study presented here is centered on the use of a biological control system in a lab setting as the means by which advanced control concepts can be taught to upper-year and graduate students within a constructivist framework. This approach was successfully applied to achieve all of the learning outcomes for the lab, but student feedback indicated that structured collaboration and metacognitive activities should have been given higher priority to improve student experiences. A re-iteration of this framework for upper-year lab curriculum design based on student feedback is presented.
\end{abstract}

Keywords: Constructivism, Synthetic Biology, Control Systems, Collaboration

\section{INTRODUCTION}

As industries require more biological knowledge of them, the demands on chemical engineering graduates are changing. Two questions arise in the face of this dynamic landscape: what biological knowledge in particular should they have? How can curricula be better designed to equip new graduates with foundational knowledge before they graduate? These questions are continually revisited at numerous universities [1].

Much of the guiding literature on the subject of teaching biology to engineering students is contentcentric: it is heavily focused on providing instructors with pre-constructed mental models for content delivery, and there has been very little exploration into the pedagogical processes by which this content could be delivered [2] These mental models are intended to connect biological concepts to engineering principles that students have already been taught in their core curriculum. For instance, Khoshmanesh et al attempt to translate the biological concept of the cell into engineering principles by constructing it as a collection of chemical processes - a familiar concept for chemical engineering students [3]. Although mental models like this may be factually accurate, they may also implicitly limit students' learning by providing pre-packaged knowledge in lieu of opportunities for personalization and ownership of new knowledge.

It may be possible to teach core engineering principles directly, with biological cases within the constructivist approach to teaching and learning [4]. In this inverted scenario, the biology must be conceptualized by students in order for them to understand the engineering. Synthetic biological control systems provide an ideal test case for this hypothesis because the control concepts they demand (feedback, time lag, model-based control) are also required to understand complex process control. Such an approach will work optimally only if students' learning is purpose-driven, and if they are allowed to direct its course. In this way, they have a reason to personalize the knowledge they construct about the concept - control systems in this case - and they have the freedom to fully develop their own mental models to describe it.

A case study based on the laboratory portion of an upper-year biomedical engineering course is presented to demonstrate these hypotheses. Due to unique circumstances, the lab was made up of a mixture of undergraduate and graduate students from two courses with related, but different curricula. This scenario created unique challenges and opportunities: how could the skillsets of these students be leveraged to maximize collaboration? How could course content be tailored to satisfy the requirements of two distinct courses? Students were asked to explore a novel biological control system about which course instructors had little practical knowledge. They were given full responsibility for its characterization, from designing the experiments they would perform to how they would be graded. 
This environment allowed them to readily conceptualize the biology required to understand the underlying control concepts: they were able to produce high-quality mathematical models which they fit to their own data and which they were able to validate using published data. They self-reported having learned about biological control systems in addition to developing several technical and soft skills. Based on the results from this case study and student feedback, a framework for scale-up to a more typical lab section size, as well as best practices are provided.

\section{COURSE CONTEXT}

\subsection{The Challenge}

BME455 - Cellular and Molecular Bioengineering II is an elective upper-year half course offered by the Institute of Biomaterials and Biomedical Engineering at the University of Toronto. It is available to undergraduate engineering students enrolled in Biomedical Engineering modules, and/or those in the Department of Chemical Engineering and Applied Chemistry at large. The lab portion of the course has historically been focused on basic techniques of mammalian cell culture and molecular biology, such as culturing and counting cells, quantifying cell adherence, and identifying/quantifying genes and proteins via PCR and SDS-PAGE.

Typically, fewer than ten students enroll in BME455 each session. In the Fall semester of 2016, the course began with an enrollment of six students, which quickly dropped down to just three participants, too few to make running the lab viable. At the same time, the instructor for BME455 was also teaching CHE1125 - Modelling and Optimization of Chemical/Biomedical Networks, a graduate level course offered by the Department of Chemical Engineering and Applied Chemistry. Typically, students in CHE1125 have a research project which makes up a portion of their grade. They were invited to join the BME455 lab in lieu of this project, with the hope that enough would accept the offer to make the lab viable.

Luckily, this was the case: three graduate students joined the lab to make a viable cohort of six. However, this posed a challenge for the laboratory instructor, as the two courses have significantly different curricula. The laboratory learning outcomes then had to fall within the overlap between the two courses, while providing adequate challenge and learning opportunities to both groups of students. Within this challenge was an opportunity to depart from the traditional course curriculum to try a new approach.

\subsection{A Solution}

Initially, one experiment involving a new, light-based control system in mammalian cells had been planned for the lab. This system was to be compared with a similar one in bacterial cells, and would have built on skills acquired in the first few laboratory sessions. However, the material planned for this experiment, involving concepts of control engineering and biological modelling, was the most relevant to both groups of students in the lab. Furthermore, the system in question had not yet been characterized by the course instructors. Therefore, there was an opportunity to allow students to work collaboratively to characterize and optimize it via iterative experimentation and modelling.

With this in mind, the lab curriculum was adjusted so that students would be taught the technical skills necessary to manipulate the system in the first three laboratory sessions. They would then spend the second half of the course using these skills to optimize the function of the system, i.e. to find parameters for which it would give a maximal output, and to be able to predict system responses to varied inputs via mathematical modelling. This was both a satisfactory solution to the challenge of mixing students from different courses and levels, and a further opportunity to understand how biological systems could be used to teach complex control concepts in an open-ended, collaborative environment.

\section{THEORETICAL FRAMEWORK AND COURSE ORGANIZATION}

Constructivist educational approaches are based on the idea of knowledge as a construction; that is, that it exists by way of experience and reflection, especially in relation to other ideas and knowledge [4]. This framework was the natural choice to inform the redesign of the BME455 lab curriculum for two reasons. First, students taking the lab came from significantly different backgrounds, and were likely to have different trajectories following its completion. It was therefore hypothesized that the best learning outcomes could be achieved if students were given space to personalize their learning, relate it to the unique body of knowledge they brought to the lab, and share this learning with their peers along the way. Second, while all labs are inherently experiential, the relatively high skill level of the mature cohort in this case allowed for a deeply open-ended approach to the lab: with just a few additional skills, they would be well-equipped to handle a complex and open problem involving biological control and optimization.

Key to constructivism are metacognitive activities, or those in which students reflect on not only what they will/have learned, but also how they will/have learned it 
[4]. Two major metacognitive activities were designed into the lab curriculum: first, groups submitted a proposal after learning the basic principles governing the control system they were to analyze, but before entering the second module of the course (i.e. what will you learn and how?); and second, following each laboratory session, students were to examine their experimental results using their models and to update their proposed work accordingly (i.e. how effective is your learning?)

Thus, the lab was re-structured to include two modules: a preparatory phase, in which students learned or refreshed their technical skills; and a project phase, in which students explored light-based control of mammalian cells in an iterative and exploratory way. In both modules, undergrads were paired with grad students because the undergraduates had some knowledge of the biology, while the graduate students were expected to have more advanced modelling skills. It was reasoned that this pairing would facilitate knowledge construction by way of the common language of math. The curriculum model is summarized in Fig 1.

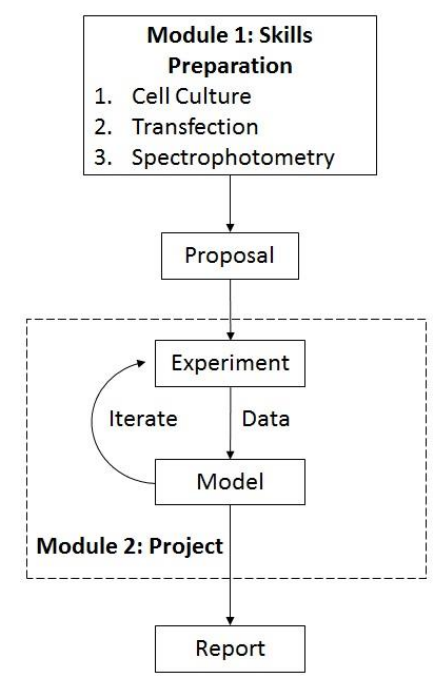

Fig. 2. Curriculum model for BME455. Following skills development, students entered the project phase via a proposal. Their experimentation informed modelling results, which allowed them to iterate on the approach they proposed.

\section{METHODS}

Due to the opportunistic nature of this study, no formal data collection or analysis was planned prior to the beginning of the course. However, informal in-lab and office hours observations were made by the lab instructor, and a learning journal survey was provided to students following submission of all graded materials. Observation of students was performed in the context of normal student-instructor relationships and was therefore not recorded, but instead used to inform general perceptions about the performance and growth of the students.

The learning journal survey asked students to rate the following dimensions on a 3-point scale in terms of both how effectively they were handled in the course and how important they were to students:

1. Course Organization

2. In-lab Organization

3. Learning Outcomes

4. Grading

5. Feedback

6. Course Content

7. Cooperation/Collaboration

Quadrant analysis was used to compare how students rated importance relative to effectiveness for each dimension. This analysis divides the total ranking space into four categories along these two dimensions. Each item is placed into one of the four quadrants based on the student responses associated with it.

The survey also gave students the opportunity to provide general feedback via three open-text questions:

\section{"Describe what you learned in this lab"}

"The structure of this lab was nontraditional. Comment on this: was it handled well? What might be done differently in the future?"

"Please provide a short reflection on your experience of the BME455 lab. Feel free to provide as much positive and critical feedback as you need to"

Thematic analysis of students' answers to these three questions was performed. Common keywords were identified across answers, grouped into themes, and ranked according to prevalence.

\section{RESULTS}

In this quadrant analysis, course dimensions which fall along diagonals can give broad insights into the relationship between student and educator perspectives. The Q1 - Q3 diagonal contains aspects for which student priorities matched educator effectiveness, while the Q2 Q4 diagonal contains aspects for which there was a disparity between student expectations and educator effectiveness. The former indicates which dimensions were implemented well and the latter indicates opportunities for improvements. 


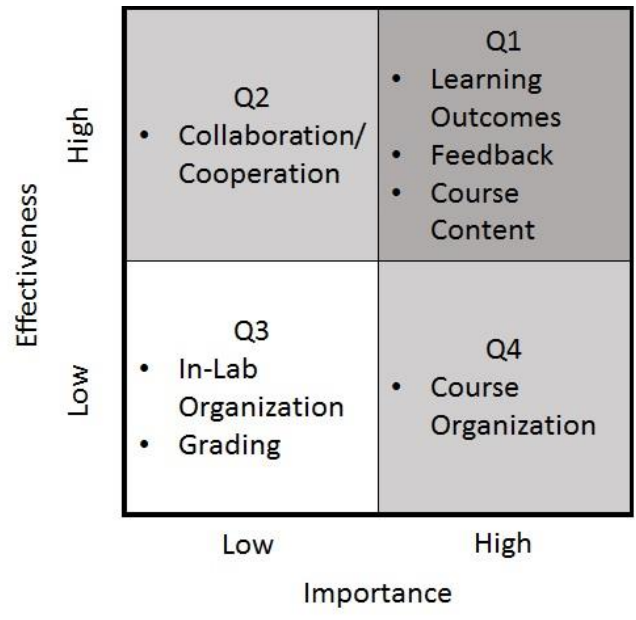

Fig. 2. Quadrant analysis of student responses to the learning journal survey.

Learning Outcomes, Feedback, Course Content, In-Lab Organization, and Grading all landed on the Q1 - Q3 axis. Thus, student expectation and educator effectiveness was aligned for these aspects of the lab. Interestingly, students indicated that learning and content were significantly more important than in-lab organization and grading. Generally, instructors give more focus to the latter than the former, but it is evidently possible that this focus could generally be better distributed to improve outcomes for students, especially those in upper-year courses.

At the end of this iteration of BME455, it was expected that students would be able to: explain the function of the control system they used; build a model describing its behavior; use experimental data to fit parameters for their model; and effectively communicate what they learned in a research paper format. As indicated in Table 1, these learning outcomes were highly aligned with the skills students independently identified as what they learned in the course. Therefore the approach used here was an effective way to meet the pre-defined learning outcomes for the lab.

Table 1: Analysis of responses to the first open-text survey question. Themes were identified across answers, then group into technical and non-technical categories. The number of times an exact phrase was provided is indicated in parentheses next to the phrase, where applicable.

\begin{tabular}{|l|l|}
\hline \multicolumn{1}{|c|}{ Technical } & \multicolumn{1}{c|}{ Non-Technical } \\
\hline Applications of & Paper-Writing (3) \\
optogenetics (2) & Experimental Design \\
Safety & Figures \\
Modelling & \\
Parameter Estimation from & \\
Experiments & \\
Toggle Switch Function & \\
Transfection & \\
\hline
\end{tabular}

Cooperation/Collaboration and Course Organization landed on the Q2 - Q4 axis, indicating that opportunities for improvement of student experience existed in these dimensions of the course. Students scored Collaboration as being of low importance (but high effectiveness), and Course Organization as being low effectiveness (but high importance). Both of these aspects were focal points of the course redesign and are central to the hypothesis that a constructivist approach to this curriculum would be most effective: student pairings were established specifically to encourage collaboration, and the course was organized specifically to give students the greatest opportunity for iterative experiential learning.

Student responses to the second open text question can provide some insight into the specific missed opportunities associated with these disparities. Relevant to the collaboration gap is the fact that students indicated the necessity for a modelling tutorial and a journal club to improve their experience in the lab. It is therefore likely that simply pairing students with different expertise was insufficient to stimulate collaboration; in fact, students indicated that they would have liked structured space for collaboration.

Table 2: Successes and opportunities identified by students in the second open-text survey question. As in Table 1, exact phrases are indicated by the number of times they appeared.

\begin{tabular}{|l|l|}
\hline \multicolumn{1}{|c|}{ Successes } & \multicolumn{1}{c|}{ Opportunities } \\
\hline Structure (2) & More lab hours (2) \\
Research Focus (2) & Student involvement in tech \\
Collaboration & development (2) \\
Freedom & Modelling tutorial \\
& More technical tasks \\
& Faster turnaround for \\
& feedback \\
& Journal Club \\
\hline
\end{tabular}

Indeed, in responses to the third question, a common theme was a lack of effective integration of the modelling component. Most students wrote that they didn't fully understand their models until it was too late to use them as communication and experimental design tools. This was meant to be the central point of collaboration between undergrads and grads, however it is obvious that insufficient organized space was provided for that to effectively be the case. 
Table 3: Summarized positive and critical feedback provided by students in the third open-text survey question.

\begin{tabular}{|l|l|}
\hline \multicolumn{1}{|c|}{ Positive Feedback } & \multicolumn{1}{|c|}{ Critical Feedback } \\
\hline Good exposure to research (2) & Better integration of \\
Enjoyed the experience, & modelling (2) \\
learned a lot & More time required \\
Taught to write concisely for & Did not understand \\
papers & toggle switch until late \\
Toggle switch was "really & in semester \\
cool" & \\
Liked flexibility & \\
Relaxed, fun environment & \\
Something like this should be & \\
done every year & \\
\hline
\end{tabular}

In terms of Course Organization, students desperately wanted more lab hours and this is likely where the gap between effectiveness and importance lies. More lab hours would have allowed them and to be involved in technical development of the light delivery system used to stimulate cells and would likely have helped to fully develop collaboration around modelling. Though adequate lab time may be hard to find, student feedback suggested that they would be open to spending time outside of the scheduled lab hours to work on their project.

\section{CONCLUSIONS AND BEST PRACTICES}

\subsection{Biological Systems Can be Used to Teach Complex Control Concepts}

Students in the BME455 lab were able to create complex control models by intelligently iterating on experiments involving an unexplored biological control system. They reported on this work in a high level, academic format. In discussing their models, they were able to use concepts such as time lag, feedback, and signal integration in qualitative ways. They achieved these learning outcomes with very little knowledge of the particular biological system at hand and just a handful of biological concepts as a starting point. Rather than beginning with a pre-packaged mental model of biological control, they were able to construct models through experiential learning and knowledge discovery as required for exploration of a biological system.

This conclusion can be extended to hypothesize that students can learn about control from highly varied contexts that go beyond block diagrams and transfer functions. Indeed, there is no agreed upon pedagogy for teaching control simulation in Chemical Engineering [5]. Biology may be an effective way to do this, even when students have little knowledge to begin with: not only does the biology provide a tangible context for understanding control, but learning about it will help prepare them to participate in a bio-based economy. Using it as a control framework will no doubt help them to construct biological knowledge in the ways which will be useful as they move beyond the control classroom.

It should be noted that these results are limited in scope due to the context of this case study. Since BME455 is an optional, upper-year course, this work can likely best inform courses of this type. Future iterations of the framework presented here will necessarily involve students of lower levels, and perhaps those in mandatory courses. Additionally, there are some key "get right" elements to this approach that should be given more focus in future study.

\subsection{Metacognitive Activities are Essential}

The first of these is the importance of metacognitive activities. In this case, though students effectively learned how to model and understand complex control systems, they did not identify control theory as a distinct learning outcome. This was likely either because the idea of "control" was too abstract relative to their concrete in-lab experiences, or because it has been framed in limited ways in their education leading up to this lab. A reflective connection to the broader concepts of control could have been useful to establish it as a key learning outcome; for example, students could have been asked to compare and contrast biological control with other control systems they had previously studied. Without these concrete connections, students may not know what they know, but the constructivist approach used here hinges on their effective assessment of the knowledge they have gained. Therefore, one failure in this case study was the insufficient number and/or scope of metacognitive activities.

\subsection{Collaboration Requires Structure}

Structured collaboration was another limiting aspect of the approach described here. Collaboration is essential to knowledge-sharing; this was highlighted by the fact that students wanted more integration of modelling and that they identified a lack of space for sharing. Optimization of a constructivist approach to this curriculum framework will require maximization of knowledge-sharing between students with different experiences. This is especially true given that an increasing number of Chemical Engineering departments offer biology-oriented majors, minors, options etc. Students taking these options have some knowledge of biology, and can share this with those who do not - provided there is space to do so. This could take place in tutorials associated with labs, as suggested by the students of BME455, but evidently assigned partnerships is insufficient. 


\section{Acknowledgments}

We acknowledge Andrey Shukalyuk for his tireless assistance in preparing experiments week-to-week.

\section{References}

[1] Varma A and Grossman IE. "Evolving trends in chemical engineering education" AIChE Journal Vol. 60, No. 11, pp 3692-3700, 2014

[2] Johnson AT (2010) Biology for Engineers. Boca Raton, FL:CRC Press, 2011 \{ISBN: 978-1-4398-9402-6\}

[3] Khoshmanesh K, Kouzani AZ, Nahavandi S, Baratchi S, Kanwar JR. "At a glance: cellular biology for engineers." Comput Biol Chem. Vol. 32, No. 5, pp 315-331, 2008

[4] Fosnot, CT Constructivism: theory, perspectives and practice. New York: Teachers College Press, 2005 [ISBN: 978-0807-77259-1\}

[5] Belton, DJ “Teaching process simulation using videoenhanced and discovery/inquiry-based learning: Methodology and analysis within a theoretical framework for skill acquisition" Education for Chemical Engineers. Vol. 17, pp 54-65, 2016

\section{APPENDIX A: PROPOSED CONSTRUCTIVIST FRAMEWORK FOR UPPER YEAR LAB DESIGN}

Based on the case study presented here, a framework for lab design is provided in Fig. 3. This framework is very similar to the one used for BME455, but includes additional metacognitive activities, such as reflecting on the planning stages following completion. In this case study, the preparatory metacognitive activity was a proposal, but it could take the form of a reflexive learning assessment (e.g. "what do/don't I know?") or the framing of an unanswered question (e.g. "can a given chemical be produced at industrial scale?") Students in BME455 used a kinetic model and non-linear least squares regression to understand their system, but models could take many other forms; the only requirement is that students be able to use them to make sense of the system at hand.

It is also suggested that the final metacognitive activity be one which extends knowledge constructed during the learning phase to other contexts. For example, students may be asked to explore how the approach they used could be applied elsewhere, or how they might use their new knowledge to build a technology.

Not shown explicitly in this framework is the role of structured collaboration space, such as tutorial time, in the iteration cycle of the learning phase.

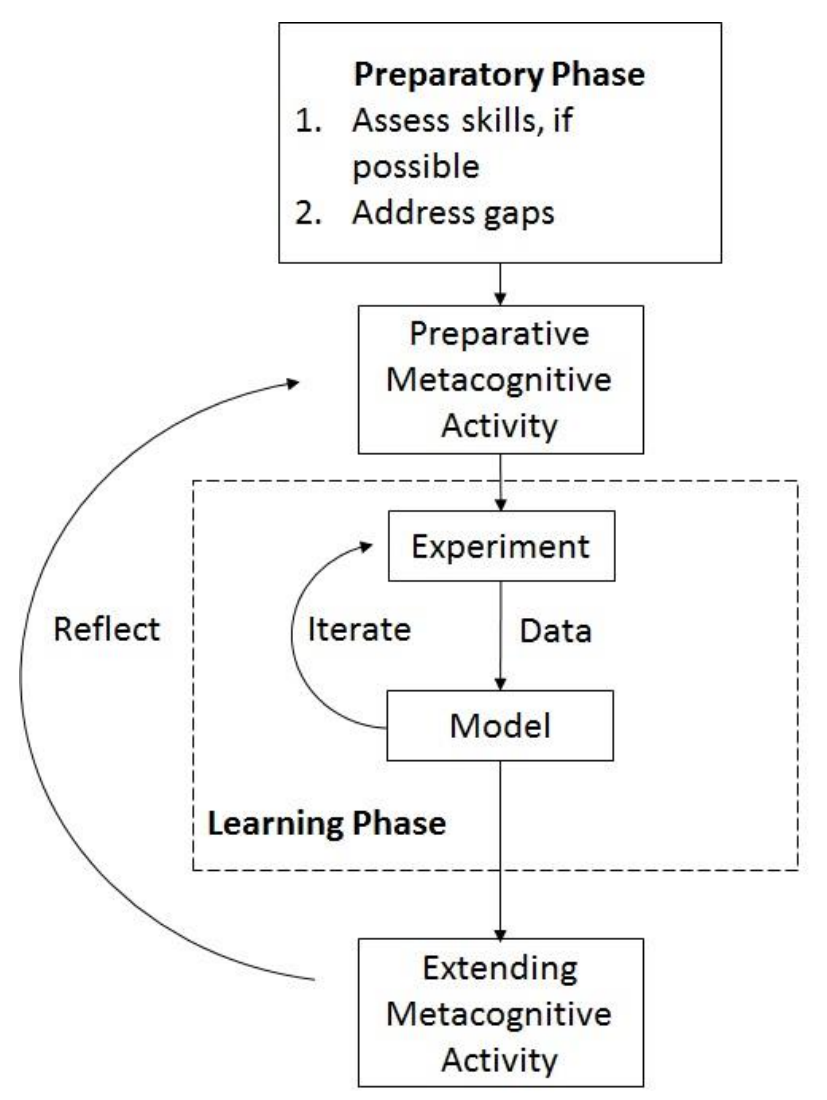

Fig. 3. Proposed framework for constructivist lab design. Skills preparation, and reflection on that preparation, is necessary for a confident approach to experimentation. Similarly to BME455, modelling should be performed to inform experimentation during the learning phase. A metacognitive activity involving reflection on the original approach, and extension of ideas to other contexts should be performed at the end of the course. 